\title{
Carnets
}

Revue électronique d'études françaises de l'APEF

Deuxième série - 2 | 2014

Frontières de la chronique

\section{Claude Crébillon, chroniqueur du libertinage chez la bonne compagnie}

Ana Alexandra Seabra De Carvalho

\section{(2) OpenEdition}

Journals

Édition électronique

URL : http://journals.openedition.org/carnets/1287

DOI : 10.4000/carnets. 1287

ISSN : 1646-7698

Éditeur

APEF

Référence électronique

Ana Alexandra Seabra De Carvalho, «Claude Crébillon, chroniqueur du libertinage chez la bonne compagnie », Carnets [En ligne], Deuxième série - 2 | 2014, mis en ligne le 30 novembre 2014, consulté le 30 avril 2019. URL : http://journals.openedition.org/carnets/1287 ; DOI : 10.4000/carnets.1287

Ce document a été généré automatiquement le 30 avril 2019.

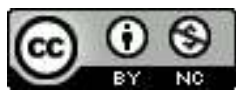

Carnets est mis à disposition selon les termes de la licence Creative Commons - Atribution - Pas d'utilisation commerciale 4.0 International. 


\title{
Claude Crébillon, chroniqueur du libertinage chez la bonne compagnie
}

\author{
Ana Alexandra Seabra De Carvalho
}

1 Le présent numéro de Carnets propose comme sujet de réflexion les «frontières de la chronique", en attirant l'attention sur le rapport entre la parole et le temps, le déplacement du réel dans l'écriture par la médiation d'un regard particulier. La chronique peut être soit la mémoire d'un passé historique glorieux, soit le panégyrique du moment présent, soit encore une peinture de l'actualité et du quotidien plutôt critique. Aux frontières de l'histoire, de la littérature et du journalisme, la chronique se présente, à mon avis, comme une forme transdisciplinaire, transgénérique et protéiforme. Je propose dans cet article une lecture des récits de Claude Crébillon (1707-1777) comme une suite de chroniques du libertinage à la mode chez «la bonne compagnie » parisienne du temps de la Régence de Philippe d'Orléans et du règne personnel de Louis XV.

2 Injustement traité de licencieux, de frivole, voire de libertin, "Girgiro, l'entortillé », épithète que l'auteur doit à la tortuosité de son style, entreprend dans tous ses récits, d'un œil fin et ironique, une sorte de voyage philosophique à travers les différents jeux de pouvoir et de séduction, en présentant à son lecteur une peinture assez vivante du libertinage mondain de son époque, dont il dévoile la fausseté dans tous ses états. En effet, le projet d'écriture de Claude Crébillon est orienté par une profonde et rigoureuse analyse du cœur humain et des masques rhétoriques de la séduction et du libertinage, c'est-à-dire, des ressorts secrets de l'amour, du désir et de la vanité, dans ses multiples variantes, tels que l'élite parisienne des premières décennies du XVIII ${ }^{e}$ siècle les met en scène (Carvalho, 2003). Il s'agit d'une belle matière à enquête, comme le remarque Johan Huizinga :

Le XVIII ${ }^{e}$ siècle, avec l'intensité de ses échanges intellectuels, encore protégés contre la surabondance chaotique par ses moyens de communication limités, devait être l'époque par excellence des joutes de plume. Celles-ci, avec la musique, la perruque, le rationalisme frivole, la grâce du rococo et le charme des salons, 
faisaient partie intégrante de ce caractère ludique général spécialement éloquent que personne n'ira contester au XVIII ${ }^{\text {e }}$ siècle et que nous lui envions quelquefois (Huizinga, 1951: 255).

La vie et l'œuvre de Claude Crébillon ont été l'objet de jugements contradictoires au cours de l'histoire littéraire française et l'épithète d'homme et d'écrivain libertin a longtemps duré. On connaît les conflits avec son père - Crébillon, le Tragique, l'honorable membre de l'Académie française. De son côté, « Le Petit » préfère la comédie italienne et le roman, genre en ascension quoique décrié par les moralistes et les critiques littéraires contemporains.

Claude Crébillon débute sa carrière d'écrivain en 1730 sous anonymat avec Le Sylphe, ou songe de Madame de $R^{* * *}$, écrit par elle-même à Madame de $S^{* * *}$ [Sy]. Il s'agit d'un texte bref mais déjà embryonnaire des questions autant thématiques que formelles que l'auteur exploitera à l'avenir. En 1732, il publie avec un très grand succès son premier roman épistolaire, les Lettres de la Marquise de $M^{* * *}$ au Comte de $R^{* * *}[L M]$, fine analyse de la passion féminine. Cependant, la parution, en 1734, de son troisième récit, L'Écumoire, ou Tanzaï et Néadarné, histoire japonaise $[T N]$ amène son emprisonnement à Vincennes pour quelques jours. L'ouvrage est condamné au bûcher, car les censeurs ont bien compris que sous le décor oriental il s'agissait plutôt d'une critique mordante à l'absolutisme royal. Dans les années suivantes, Crébillon écrit quelques-uns de ses récits les plus connus: Les Égarements du cœur et de l'esprit ou Mémoires de M. de Meilcour [Ég] (1736-1738) ; Le Sopha, conte moral [So], qui n'est publié qu'en 1742, mais dont le parfum oriental n'arrive pas à cacher la critique de la monarchie française contemporaine : cette critique vaut à l'auteur récidiviste une condamnation d'exil. Il écrit probablement aussi une première version des deux dialogues La Nuit et le Moment, ou les matines de Cythère [NM] (publié en 1755) et Le Hasard du coin du feu, dialogue moral [HCF] (publié seulement en 1763), où les affaires du cœur et des sens, tout comme dans le Sylphe et les Égarements, sont traités avec beaucoup d'esprit. En 1746, voit le jour un récit intitulé Les Amours de Zéokinizul, roi des Kofirans, texte dont la paternité reste assez douteuse. En effet, la critique en est divisée: les uns l'attribuent à Crébillon à cause du nom anagrammatique de l'auteur supposé (Krinelbol) et d'une réédition postérieure qui présente une clé des noms réels. Les autres l'attribuent soit à La Beaumelle soit à un écrivain inconnu. Toutefois, cet ouvrage satirique me semble trop direct et transparent, ce qui s'éloigne beaucoup du style gazé de "Girgiro, l'entortillé ». En 1754, paraissent Les Heureux Orphelins, histoire imitée de l'Anglais [HO] (transposition dans la cour anglaise du libertinage à la française) et les sept premières parties d'Ah quel conte ! conte politique et astronomique [AQC], terminé l'année suivante et qui peut être lu comme une suite au Sopha. En 1755, paraît aussi La Nuit et le Moment. À cette époque Crébillon a probablement été le secrétaire du duc de Richelieu et, en 1759, il devient « censeur royal pour les belles-lettres ", comme son père auparavant. Le Hasard du coin $\mathrm{du}$ feu paraît finalement en 1763. Sans avoir eu l'honneur d'appartenir comme son père à l'Académie française, Claude Crébillon, en 1766, est néanmoins reçu comme membre non résident à l'Académie des Sciences, des Arts et des Belles-Lettres de Dijon. L'auteur publie, en 1768, sa seconde monodie épistolaire, les Lettres de la Duchesse de ${ }^{* * *}$ au Duc de ${ }^{* * *}[L D]$, où il développe encore une fois l'analyse du cœur féminin. En 1771, paraît son dernier ouvrage, les Lettres athéniennes, extraites du portefeuille d'Alcibiade [LA], encore un roman épistolaire, mais à plusieurs voix, où le contexte de la Grèce de Périclès miroite à nouveau " la bonne compagnie " libertine de la France du XVIII ${ }^{e}$ siècle. Six années plus tard, le 12 avril 1777, survient la mort de Claude Prosper Jolyot de Crébillon, «Écuyer, Censeur royal ». 
Crébillon a été un des écrivains les plus lus de son époque. Ce succès auprès du public est attesté par les éloges qu'un bon nombre de critiques et d'auteurs lui accordent, comme par exemple La Morlière, D'Alembert, Baculard d'Arnauld, Du Coudray, La Chesnaye des Bois, Voltaire, Palissot, Collé, Saurin, etc. Cependant, il y a aussi ceux, tels que Diderot, Grimm ou Sade, qui trouvent ses ouvrages trop frivoles et licencieux, surtout les textes publiés entre 1754 et 1771, jugés ennuyeux et entortillés. Ces dernières critiques sont fort injustes, à mon avis, car quand on lit ses récits en profondeur, on aperçoit plutôt le regard fin et ironique du chroniqueur des mœurs corrompues de son époque, sa fine analyse psychologique, sociologique et même politique portée sur les différents jeux de pouvoir et de séduction, dont il dévoile les illusions, les faussetés, les ruses, les cruautés. En plus, il est maitre d'un style raffiné qui se montre le plus adéquat soit au jargon de «la bonne compagnie ", soit à la subtilité de son enquête sur les mœurs de ce cercle clos. Il faut aussi souligner la recherche esthétique menée par l'auteur sur les potentialités et les frontières du récit, notamment du roman.

À côté de l'exploit sur les possibles romanesques, l'amour dans tous ses états est le sujet de tous les récits de Claude Crébillon. L'auteur y analyse les ressorts secrets de la passion, du désir, du libertinage et de la vanité, dans ses multiples variantes («c'est toujours l'amour que l'on voit sous des formes différentes »- Crébillon, 1999 [Lettres de la Marquise ]: 80). Thématique intemporelle, bien sûr, elle est pourtant passible d'actualisations fort différentes selon les époques. La littérature est, en effet, un moyen privilégié de miroiter, véhiculer et produire les idéaux et les idées régnants dans un cadre spatio-temporel donné, aussi bien que les changements opérés à l'intérieur d'une certaine époque. En ce qui concerne le domaine français, Nina Epton (1963) dégage schématiquement quatre systèmes conceptuels de l'amour jusqu'au XVIII siècle : l'amour courtois du Moyen Âge, l'amour galant de la Renaissance (époque caractérisée par son paganisme et son épicurisme - à côté, paradoxalement, du néoplatonisme), l'amour précieux du XVII ${ }^{\mathrm{e}}$ siècle (partagé entre deux tendances : l'héroïsme, d'un côté, et le cynisme, de l'autre) et l'amour libertin dix-huitièmiste. Ce dernier est censé se caractériser par le culte de l'élégance cynique et de la sensualité. D'ailleurs, les frères Edmond et Jules de Goncourt, deux célèbres auteurs du XIX $\mathrm{X}^{\mathrm{e}}$ siècle, avaient déjà opposé la conception amoureuse du Grand Siècle et celle du dix-huitième de la façon suivante :

Jusqu'à la mort de Louis XIV, la France semble travailler à diviniser l'amour. Elle fait de l'amour une passion théorique, un dogme entouré d'une adoration qui ressemble à un culte. Elle lui attribue une langue sacrée qui a les raffinements de formules de ces idiomes qu'inventent ou s'approprient les dévotions rigides, ferventes et pleines de pratiques. Elle cache la matérialité de l'amour avec l'immatérialité du sentiment, le corps du dieu avec son âme. Jusqu'au dix-huitième siècle, l'amour parle, il s'empresse, il se déclare, comme s'il tenait à peine aux sens et comme s'il était, dans l'homme et dans la femme, une vertu de grandeur et de générosité, de courage et de délicatesse. Il exige toutes les épreuves et toutes les décences de la galanterie, l'application à plaire, les soins, la longue volonté, le patient effort, les respects, les serments, la reconnaissance, la discrétion. Il veut des prières qui implorent et des agenouillements qui remercient, et il entoure ses faiblesses de tant de convenances apparentes, ses plus grands scandales d'un tel air de majesté, que ses fautes, ses hontes même, gardent une politesse et une excuse, presque une pudeur. Un idéal, dans ces siècles, élève à lui l'amour, idéal transmis par la chevalerie au bel esprit de la France, idéal d'héroïsme devenu un idéal de noblesse. Mais au dix-huitième siècle que devient cet idéal ? L'idéal de l'amour au temps de Louis XV n'est plus rien que le désir, et l'amour est la volupté (Goncourt, 1877 : 150-151). 
7 On peut nettement reconnaître dans cet extrait les clichés qui caractérisent la question érotique aux $\mathrm{XVII}^{\mathrm{e}}$ et $\mathrm{XVIII}^{\mathrm{e}}$ siècles (avant Rousseau). On peut donc y déceler deux systèmes divergents, du moins en apparence, correspondants, grosso modo, à un système de la vertu (l'héroïsme passionnel) et à un autre, celui du désir libertin. On vérifie, d'après les auteurs cités, que, dans le premier cas, l'amour est un concept à la fois mythifié et mystifié. Idéal divinisé, théorie/dogme, concrétisé par la parole/langue sacrée plutôt que par les sens, l'âme qui cache le corps, il exige des qualités et des mérites moraux fondés sur la vertu (grandeur, générosité), dont les épreuves (plaire, les soins, les serments, les respects, la reconnaissance, la discrétion, les prières, les remerciements, la politesse, la majesté, l'excuse, etc.) rendent l'individu aimable/adorable, en dignifiant les amants et l'amour. Cette conception de l'amour-passion puise aux sources d'une tradition occidentale ancienne (le XII ${ }^{\mathrm{e}}$ siècle, selon Denis de Rougemont dans son célèbre essai intitulé L'Amour et l'Occident - cf. Rougemont, 1939) qui définit l'amour comme une force toute-puissante et anéantissante, sur laquelle le sujet passionnel - en réalité, plutôt objet/victime - ne peut avoir aucun contrôle conscient et dont il sera fatalement accablé. Néanmoins, l'aspect inconscient, involontaire du sentiment amoureux donne naissance à deux positions divergentes face à la valeur - positive ou négative - qu'il peut prendre. D'une part, la position positive correspond au courant représenté par le roman héroïque, préclassique, où le happy ending - le mariage des amants - constitue le retour à l'ordre social, victoire heureuse de l'alliance du sentiment et de la raison sur la fatalité du mythe. Par contre, la perspective classique envisage la passion sous l'angle du danger : l'intégrité unitaire du sujet est mise en péril, déchirée par l'excès de l'intensité des sentiments (cf. Kibédi-Varga, 1963 : 972). Si le bouleversement profond de l'être touché par la passion est opéré par cette force extérieure à sa conscience et à sa volonté, il doit alors, pour rétablir son équilibre, son repos, en prendre connaissance - se comprendre - et, ensuite, la refuser dans un effort de résolution de son dilemme intérieur, issu de l'opposition irréconciliable, établie par le code de la bienséance, entre vertu (devoir) et passion. Le fait que l'obstacle soit imposé par un système, parfaitement intériorisé, de règles de conduite contradictoires - sévérité de la vertu et futilité des agréments et des plaisirs de la vie mondaine -, plutôt que par le sujet lui-même, en conformité avec ses valeurs religieuses, morales, etc., entraîne le déchirement douloureux et honteux, aussi bien que le remords, suivis d'une illusion de solution - la mort, réelle ou symbolique, qui lui permettra la pérennité du sentiment malgré l'interdit social.

8 Claude Crébillon se connaît bien dans cette tradition littéraire, culturelle et sociologique. En plus, il observe attentivement ses contemporains et décide alors de faire la chronique de cette Gaule amoureuse, galante et libertine dans tous ses récits. En effet, tout comme son personnage Chester (mais d'un point de vue moral opposé), l'auteur pourrait affirmer : « moi, c'est le cœur que je développe » (Crébillon, 2001 [HO] : 180). Pourtant, par delà les illusions du coup de foudre et de l'amour-passion ce sont surtout les masques de l'amour-goût (le simple désir) qui intéressent l'auteur. Effectivement, comme le remarque Philip Stewart, à la mort de Louis XIV, « les masques (...) ne sont pas censés tromper mais permettre une forme conventionnelle et stable du paraître » (Stewart, 1973 : 88). C'est-àdire que, pendant la Régence et le règne personnel de Louis XV, on affiche la sensualité, pourtant sous une forme cyniquement décente et acceptable, celle du système du libertinage galant. Les personnages crébilloniens appartiennent tous à ce cercle clos de l'aristocratie parisienne. Les uns se connaissent parfaitement dans les règles strictes des jeux galants et du jargon à la mode, soit qu'ils jouent le jeu de la séduction pour satisfaire 
leurs sens, leurs cœurs ou leurs esprits, soit qu'ils s'intéressent plutôt à exercer leur pouvoir de domination libertine sur les autres. Ceux-ci sont des néophytes naïfs ou des jeunes femmes passionnées qui deviennent facilement les proies des libertins. D'ailleurs, Jacques Rustin note que le libertinage est aussi « une discipline exercée sur soi-même » (Rustin, 1979 : 101), ayant pour but l'affirmation du désir, du plaisir et du bonheur de l'individu. L'évolution sociale, vérifiée dans les mœurs et dans les rapports entre les sexes, est, on le sait, la conséquence des idées nouvelles sur la liberté et le bonheur répandues par les philosophes ${ }^{1}$ et imprégnées, à son tour, des théories des sciences naturelles et physiques. En réalité, on essaie d'adopter ces concepts dans le champ des relations amoureuses, de façon à surpasser l'opposition entre la vertu et la passion par la conciliation des contraires. Clitandre, par exemple, dans La Nuit et le Moment, tel un physicien qui fait l'analyse des sophismes amoureux, se sert d'un langage mécanicien pour vérifier si « la machine l'emporte sur le sentiment » (Crébillon, $2000[N M]: 610)$. Ce joyeux libertin considère son époque fort plaisante et « délicieu[se] à considérer un peu philosophiquement»(552), car, selon lui, «jamais les femmes n'ont mis moins de grimaces dans la société ; jamais l'on n'a moins affecté la vertu » (541). Et il ajoute :

On se plaît, on se prend. S'ennuie-t-on l'un avec l'autre? on se quitte avec tout aussi peu de cérémonie que l'on s'est pris. Revient-on à se plaire? on se reprend avec autant de vivacité que si c'était la première fois qu'on s'engageât ensemble. On se quitte encore, et jamais on ne se brouille. Il est vrai que l'amour n'est entré pour rien dans tout cela; mais l'amour, qu'était-il qu'un désir que l'on se plaisait à s'exagérer, un mouvement des sens, dont il avait plu à la vanité des hommes de faire une vertu? On sait aujourd'hui que le goût seul existe; et si l'on se dit encore qu'on s'aime, c'est bien moins parce qu'on le croit, que parce que c'est une façon plus polie de se demander réciproquement ce dont on sent qu'on a besoin. Comme on s'est pris sans s'aimer, on se sépare sans se haïr, et l'on retire du moins, du faible goût que l'on s'est mutuellement inspiré, l'avantage d'être toujours prêts à s'obliger (...) et je crois, à tout prendre, qu'il y a bien de la sagesse à sacrifier à tant de plaisirs quelques vieux préjugés qui rapportent assez peu d'estime, et beaucoup d'ennui à ceux qui en font encore la règle de leur conduite (Crébillon, 2000 [NM] : 541-542).

C'est vraiment une magnifique synthèse du «vice à la mode» (Rustin, 1979) dans le monde aristocratique français $\mathrm{du} \mathrm{XVIII}^{\mathrm{e}}$ siècle, qui est un microcosme sociable et raisonnable par excellence, et dont Claude Crébillon se fait le chroniqueur par l'intermède de ses personnages, soit qu'ils l'approuvent ou qu'ils le condamnent. L'écrivain nous donne à lire, apparemment sans prendre aucun parti, l'épuisement du sens moral de concepts-clé tels que bienséance, qui ne devient pour ses personnages qu'un synonyme de décence, ou vertu, maintenant rien d'autre que simple savoir-faire ou savoir-vivre mondain, car il ne s'agit plus d'une qualité intérieure mais d'un rôle bien joué. Alors, tous les récits de l'auteur, qu'ils se déroulent à son époque ${ }^{2}$ ou que l'écrivain les transpose dans un ailleurs féerique ou historique ${ }^{3}$, rendent compte d'un groupe social mené par le penchant sensuel de la passion et du désir, comme recherche et de la liberté et du bonheur de l'individu. On remplace donc la passion par le seul désir : le plaisir devient le nouvel idéal et les mérites ne sont plus d'ordre moral mais intellectuels, physiques et sociables (politesse, galanterie, science des voluptés).

10 L'ambiance sociale dans les récits de Claude Crébillon est marquée par l'oisiveté, laquelle semble entraîner fatalement l'ennui des personnages. Afin de combattre ce terrible ennemi, ceux-ci essaient de prendre toutes les armes dont ils peuvent disposer, et alors la vie de cette société parasite s'anime de fêtes somptueuses, de spectacles magnifiques, de jeux divertissants, de galanteries joyeuses. Dans Tanzaï et Néadarné, l'auteur donne 
justement un inventaire détaillé, non sans une très fine ironie, des talents les plus appréciés dans la cour de l'île Babiole, exemple de celle de la France de son temps :

[Cormoran] était le plus beau danseur du monde, personne ne faisait la révérence de meilleure grâce, il devinait toutes les énigmes, jouait bien tous les jeux, tant de force que d'adresse, depuis le Trou-Madame jusques au Ballon. Sa figure était charmante, et empaquetée, si l'on peut le dire, dans les agréments les plus rares; il savait accompagner de toutes sortes d'instruments une voix charmante qu'il avait. (...) Outre les talents que je viens de nombrer, continua-t-elle [la fée Moustache], il faisait joliment des vers; sa conversation enjouée et sérieuse, satisfaisait également par ses grâces et sa solidité. Austère avec la Prude, libre avec la Coquette, mélancolique avec la Tendre, il n'y avait pas une Dame à la Cour dont il ne fit les délices, et pas un homme dont il ne créât la jalousie. La supériorité de son esprit ne le rendait pas insociable, complaisant avec finesse, il savait se plier à tout; il possédait mieux que personne ce langage brillant de notre Île ; il n'y avait personne qui ne fût comblé de l'entendre, et quoique cet être farouche, intitulé le bon sens, n'agit pas toujours civilement avec ce qu'il disait, l'élégance insoutenable de ses discours faisait qu'il n'y perdait rien, ou que le bon sens, caché derrière une multitude miraculeuse de mots placés au mieux, aurait paru d'une insipidité affadissante à ses Sectateurs les plus absurdes, s'il eût été vêtu moins légèrement (Crébillon, 1999 [TN] : 351-352).

11 En chroniqueur fidèle de son époque bavarde, Crébillon nous montre que, pour bien réussir dans le cercle clos de l'aristocratie française, il faut être un brillant causeur, car c'est une des plus importantes qualités qui donnent du mérite à un individu, à côté de la grâce de ses manières et de l'élégance de sa parure. En effet, en développant un goût très vif pour l'assemblage mondain, la «bonne compagnie» crée ainsi les conditions favorables à l'épanouissement de l'art de la conversation, dont Crébillon, dans tous ses ouvrages, analyse avec beaucoup de finesse les règles linguistiques, rhétoriques et bienséantes. Le principal but de l'auteur est justement de mettre en évidence comment ces règles-là sont établies, connues et acceptées par tous les intervenants. Bien sûr, ces règles peuvent ne pas être respectées, à condition qu'on le dissimule habilement aux yeux du public, puisque tout éclat a comme conséquence la punition de l'infracteur, et parfois celui-ci doit même être chassé de ce cercle clos pour que le difficile équilibre interne puisse se tenir (souvenons-nous de l'épisode de Zulica dans Le Sopha, par exemple). Chez Crébillon, on s'aperçoit donc que dans le monde règne le paraitre et par conséquent les personnages intelligents savent qu'ils doivent toujours jouer selon les règles, afin de ne pas perdre leur respectabilité et leur pouvoir (les libertins Versac, dans Les Égarements, ou Alcibiade, dans Les Lettres athéniennes, l'expliquent nettement à leurs prosélytes).

Pour les moralistes du XVII e siècle, on le sait bien, l'honnêteté était le pilier fondamental du caractère de l'individu et même quand on ne l'était pas, il fallait absolument le paraitre. La bienséance, en tant que code moral et social, était devenue presque une institution régulatrice des mœurs mondaines. Au siècle suivant, pendant la Régence et le règne de Louis $\mathrm{XV}$, les choses changent. Les personnages crébilloniens témoignent du débordement absolu et public des règles sévères de conduite imposées dans la cour du feu roi. Pourtant, cela n'abolit pas la nécessité d'un système de règles quel qu'il soit, et l'auteur nous montre qu'en réalité on affiche gaiement une conduite plutôt libertine qui veut arracher les masques de la fausse vertu, car, d'après Jacques Rustin, « l'assentiment au vice dans la première partie du XVIII ${ }^{e}$ siècle s'affirme comme une preuve d'honnêteté et de lucidité raisonnée, comme le refus des masques honteux du siècle précédent » (Rustin, 1979: 76). Alors, dans le cercle aristocratique dont Crébillon fait la chronique la 
vertu n'est plus qu'une chimère, un préjugé ridicule, un masque affiché. Comme l'affirme Nassès, dans Le Sopha :

Je le vois dans le monde (...); qu'y cherchons-nous? L'amour? Non, sans doute. Nous voulons satisfaire notre vanité, faire sans cesse parler de nous, passer de femme en femme, pour n'en pas manquer une, courir après les conquêtes, même les plus méprisables : plus vains d'en avoir eu un certain nombre, que de n'en posséder qu'une digne de plaire ; les chercher sans cesse, et ne les aimer jamais (Crébillon, $2000[\mathrm{So}]:$ :23).

Le galant homme brillant du Grand Siècle devient maintenant l'homme à bonnes fortunes, le petit-maitre fat, frivole et ridicule, dont les talents ne servent qu'à se faire une belle réputation par la glorieuse liste de ses conquêtes amoureuses laquelle lui donne un éclat incomparable dans le grand monde, le plaçant à la mode. Rappelons-nous des personnages suivants : le Sylphe (Sy), Jonquille (TN), Mazulhim et Nassès (So), Clitandre ( NM) ou Clerval (HCF), parmi d'autres. Pourtant, il y a aussi des femmes aux mœurs semblables : Célie (HCF), Zulica (So), Mme de Senanges (Ég), Mme de Rindsey (HO), Némée ( $L A)$, la fée Tout-ou-rien et la Reine des Isles de Chrystal ( $A Q C)$, etc..

14 À côté de la chronique d'un microcosme social frivole et galant, les œuvres de Claude Crébillon nous présentent encore la chronique détaillée et ironique des manœuvres plus lucides et cruelles mises en marche par quelques personnages libertins contre des êtres naïfs ou amoureux. Parmi eux, se détachent Versac (Ég), Chester (HO) et Alcibiade (LA). Ils agissent à la manière sophiste, par le jeu rhétorique de la parole séductrice, tout en décevant, en intimidant, en conquérant et en humiliant l'amour-propre de la victime, victoire ambitionnée par ces libertins cyniques, brillants stratèges et tacticiens du jeu de la séduction, qui, à cette maîtrise performative, allient encore un système analytique et théorisant. Mus par leur amour-propre et/ou par une cruauté méthodique, ces maîtres libertins reconnaissent et acceptent l'immoralité généralisée, maîtrisent avec lucidité les règles conventionnées du jeu qu'ils acceptent de jouer, mais ils ont aussi la capacité de s'en distancier par la réflexion. En conséquence, ils croient encore à la supériorité de leur savoir, ce qu'ils appellent la science du monde, et à leur fonction didactique, en devenant des maitres à penser de quelques disciples élus. Versac (Ég), par exemple, explique à son disciple comment on doit agir dans le monde :

Pensez-vous que je me sois condamné sans réflexion au tourment de me déguiser sans cesse ? Entré de bonne heure dans le monde, j'en saisis aisément le faux. J'y vis les qualités solides proscrites, ou du moins ridiculisées, et les femmes, seuls juges de notre mérite, ne nous en trouver qu'autant que nous nous formions sur leurs idées. sûr que je ne pourrais, sans me perdre, vouloir résister au torrent, je le suivis. Je sacrifiai tout au frivole; je devins étourdi pour paraître plus brillant, enfin, je me créai les vices dont j'avais besoin pour plaire : une conduite si ménagée me réussit (Crébillon, $2001[E ́ g]$ : 214).

15 Crébillon montre, dans tous ses récits, que le libertinage, à ce niveau supérieur, c'est un exercice de maîtrise de soi et des autres, où le désir érotique est remplacé par le désir cérébral de pouvoir sur soi-même, sur les femmes (la guerre des sexes), sur le social (la satisfaction de l'amour-propre et, dans certains cas, l'ambition politique). Selon Colette Cazenobe, ces libertins cherchent inlassablement

la vivacité, la multiplicité des jouissances sensuelles, une réputation de séducteur au-dessus de tout soupçon, la satisfaction de l'emporter sur tous par ruse, expérience et sang-froid, le plaisir de manœuvrer, d'attaquer et de rompre, la joie subtile d'humilier la partenaire, de la perdre aux yeux du monde et de la réduire à merci de toutes les manières, par les sens, par le cœur et par l'esprit ... Tous ces 
vœux font partie d'une sorte d'idéal de libertinage dont Crébillon a rassemblé ou accusé plus qu'inventé les traits généreux. Mais il a fait plus que de les composer entre eux. Il les a fait concourir à l'avènement d'un libertinage encore inédit quand à sa visée et sa plénitude, d'un libertinage tout à la fois esthétique et théoricien, réfléchi, proclamé, voulu et pratiqué artistement pour lui-même. Le libertinage d'Alcibiade est un choix de vie (...). Désormais, le dessein essentiel du "grand » libertin sera précisément de l'être à la perfection, sans réserve ni retour, en toute liberté comme en toute conscience et avec un beau souci d'exemplarité ; il sera en outre de faire du libertinage lui-même un art de société, brillant et dangereux, l'art de jouer sa liberté contre celle des autres et de gagner à cette sorte de jeu sensuel et bavard par les seules grâces de l'esprit et les puissances de la séduction (Cazenobe, 1997 : 31-32).

16 À côté de l'analyse en profondeur des mœurs corrompues de «la bonne compagnie ", Crébillon analyse aussi avec beaucoup de finesse son jargon amoureux, à fin de mettre à nu les masques d'Eros. Tous les personnages de Crébillon parlent le jargon à la mode, soit qu'ils y croient, soit qu'ils feignent d'y croire. Pourtant, les libertins usent ce langage équivoque pour établir un empire de la liberté et de la volonté personnelle, en transgressant les lois fondatrices de la société. C'est pourquoi le dialogue - en présence ou par lettres - occupe une place si importante dans toutes les œuvres de l'auteur. C'est que la civilité se joue aussi au niveau du discours amoureux avec ce ton de la bonne compagnie. Selon Versac, il consiste dans

une négligence dans le maintien, qui, chez les femmes, aille jusques à l'indécence, et passe chez nous ce qu'on appelle aisance, et liberté. Tons et manières affectés, soit dans la vivacité, soit dans la langueur. L'esprit frivole et méchant, un discours entortillé, voilà ce qui, ou je me trompe fort, compose aujourd'hui le ton de la bonne compagnie (Crébillon, $2001[E ́ g]$ : 218).

Ce langage équivoque est un voile de décence qui sert à embellir le libertinage des mœurs pour les protéger du chaos et de l'exposition publique dégradante. Comme le remarque Colette Cazenobe :

l'héritage de l'honnêteté se maintient dans une politesse exquise, des façons parfaites, un langage qui raffine encore sur les exigences de tenue et de distinction acquises à l'école de la préciosité et des maîtres du siècle précédent, tels Méré et Saint-Évremond ; mais, s'il y a continuité, il y a aussi rupture : il ne s'agit plus de rendre la vertu traitable, il est question de rendre le vice aimable. À mesure que les mœurs se gâtent, les propos se font plus délicats pour parvenir à exprimer, dans une harmonie inégalable et inégalée, les secrets des cœurs corrompus (Cazenobe, $1991: 32-33$ ).

Pour conclure, on peut dire que Claude Crébillon se fait le chroniqueur et des mœurs corrompues de la bonne compagnie et de son jargon amoureux. Pourtant, au contraire de son père, il se donne à exploit des possibles narratifs dans le roman, genre en ascension à son époque malgré les critiques sévères des moralistes et des critiques littéraires. Son œuvre peut bien être lue comme une chronique fidèle du microcosme social de l'aristocratie parisienne dix-huitièmiste, dont il dévoile l'hypocrisie et le libertinage. Pour ce faire, l'auteur entreprend, d'un côté, une expérimentation des possibles discursifs des stratégies et des tactiques du jargon amoureux (faux ou sincère) - d'où la pertinence de son style gazé, allusif et tortueux; mais, de l'autre côté, il s'aventure aussi dans l'expérimentation des possibles narratifs, en exploitant les modes dictées par le public et l'immense plasticité des différentes formes narratives. De cette façon, Crébillon a largement contribué à la problématisation des conventions poétiques et rhétoriques de son temps, dans une attitude de grande modernité qui n'a été reconnue qu'au $\mathrm{XX}^{\mathrm{e}}$ siècle, 
qui le rachète des «Enfers » des bibliothèques. Cette modernité permet au lecteur de nos jours de se délecter non pas avec de simples récits érotiques, mais avec une suite de chroniques qui analysent finement et de façon non engagée les stratégies et les tactiques de l'escrime argumentative et linguistique du jargon amoureux de l'aristocratie libertine de son époque, en appelant beaucoup plus au plaisir intellectuel et esthétique qu'à la jouissance érotique. Comme l'affirme Jean Dagen :

La véritable réhabilitation de Crébillon consiste non pas à faire de lui le plus brillant représentant du roman libertin au XVIII siècle ou l'avocat de la liberté sexuelle, mais à traiter ses romans comme les livres d'un maître du genre, celui qui à son époque a le mieux perçu et illustré l'exigence de rigueur que le roman devait s'imposer, le mieux interprété la relation de l'esthétique de la prose avec une pensée qui réunisse la vision de l'homme et la conception de l'art sous le signe de l'artifice (Dagen, 1995 : 26-27).

\section{BIBLIOGRAPHIE}

BAUDRILLARD, Jean (1994). Les stratégies fatales. Paris : Le Livre de Poche.

BRABANT, Roger (1994). « La machine infernale ou le jeu de l'amour selon Crébillon fils », Revue d' Histoire Littéraire de la France, nํ2, pp. 198-220.

CARVAlho, Ana Alexandra Seabra de (2003). O Jogo do desejo em Claude Crébillon. Estudo dos processos retóricos da sedução. Lisboa : Fundação Calouste Gulbenkian / Fundação para a Ciência e a Tecnologia.

CARVAlHo, Ana Alexandra Seabra de (2012). «Sentidos da extravagância dos contos em Le Sopha e Ah Quel Conte! de Claude Crébillon » [on-line], Carnets IV, (Res)sources de l'extravagance, janvier 2012, pp. 63-84.

<URL : http://ler.letras.up.pt/uploads/ficheiros/12137.pdf>.

CAZENOBE, Colette (1991). Le système du libertinage de Crébillon à Laclos. Oxford : The Voltaire Foundation.

CAZENOBE, Colette (1997). Crébillon fils ou la politique dans le boudoir. Paris-Genève : Éd. Honoré Champion.

CITTON, Yves (1994). Impuissances : Défaillances masculines et pouvoir politique de Montaigne à Stendhal. Paris : Aubier.

CONROY, Peter (1972). Crébillon fils: Techniques of the Novel. Oxford : Voltaire Foundation.

CRÉBILLON, Claude (1999). CEuvres complètes I (éd. dir. par Jean Sgard : Le Sylphe, ou songe de Madame de $R^{* * *}$ écrit par elle-même à Madame de $S^{* * *}$ [éd. critique par Carmen Ramirez] ; Lettres de la Marquise de $M^{* * *}$ au Comte de $R^{* * *}$ [éd. critique par Suzanne Cornand] ; Tanzaï et Néadarné, histoire japonaise [éd. critique par Jean Sgard] ; Les Amours de Zéokinizul, roi des Kofirans, ouvrage traduit de l'Arabe du voyageur Krinelbol [éd. critique par Simon F. Davies]). Paris : Classiques Garnier Multimédia. 
CRÉBILLON, Claude (2000). Euvres complètes II (éd. dir. par Jean Sgard : Les Égarements du cour et de l'esprit, ou Mémoires de M. de Meilcour [éd. critique par Michel Gilot et Jacques Rustin] ; Le Sopha, conte moral [éd. critique par Jean Sgard] ; Dialogues des Morts [éd. critique par Aurelio Principato] ; La Nuit et le Moment ou les Matines de Cythère, dialogue [éd. critique par Jean Oudart] ; Le Hasard du coin $\mathrm{du} f e u$, dialogue moral [éd. critique par Marie-Françoise Luna et Jean Oudart]). Paris : Classiques Garnier Multimédia.

CRÉBILLON, Claude (2001). CEuvres complètes III (éd. dir. par Jean Sgard : Les Heureux Orphelins, histoire imitée de l'Anglais [éd. critique par Philip Stewart] ; Ah quel conte ! conte politique et astronomique [éd. critique par Régine Jomand-Baudry]). Paris : Classiques Garnier Multimédia.

CRÉBILLON, Claude (2002). CEuvres complètes IV (éd. dir. par Jean Sgard : Lettres de la Duchesse de*** au Duc de*** [éd. critique par Bernadette Fort] ; Lettres athéniennes, extraites du portefeuille d'Alcibiade [éd. critique par Véronique Costa et Dominique Holzle]). Paris : Classiques Garnier Multimédia. DAGEN, Jean (1995). Introduction à la sophistique amoureuse dans Les Égarements du cœur et de l'esprit de Crébillon fils. Paris : Honoré Champion.

DORNIER, Carole (1994). Le discours de maîtrise du libertin : Étude sur l'œuvre de Crébillon fils. Paris : Klincksieck.

ELIAS, Norbert (1985). La Société de Cour. Paris : Flammarion (1969).

EPTON, Nina (1963). Histoire de l'amour en France. Paris : Hachette (1959).

FORT, Bernadette (1978). Le langage de l'ambiguïté dans l'œuvre de Crébillon fils. Paris : Klincksieck.

FUNKE, Hans-Günter (1972). Crébillon fils als Moralist und Gesellschaftskritiker. Heidelberg : Carl Winter Universitätsverlag.

GÉRAUD, Violaine (1995). La lettre et l'esprit de Crébillon fils. Paris : SEDES.

GIARD, Anne (1986). Savoir et récit chez Crébillon fils. Paris-Genève : Champion-Slatkine.

GONCOURT, Edmond et Jules de (1877). La femme au dix-huitième siècle. Paris : G. Charpentier \& Cie (1862).

HARTMANN, Pierre (1998). Le contrat et la séduction : Essai sur la subjectivité amoureuse dans le roman des Lumières. Paris : Honoré Champion.

HUIZINGA, Johan (1951). Homo ludens. Essai sur la fonction sociale du jeu. Paris : Gallimard (1938).

JOSEPH, Jean René (1984). Crébillon fils : économie érotique et narrative. Lexington, Kentucky : French Forum Publisher.

KIBÉDI-VARGA, Aron (1963). « La désagrégation de l'idéal classique dans le roman français de la première moitié du XVIII ${ }^{\mathrm{e}}$ siècle ", Studies on Voltaire and the Eighteenth Century, vol. 26, Oxford : The Voltaire Foundation.

REY, Alain (dir.) (1992). Dictionnaire historique de la langue française. Paris : Dictionnaires Le Robert. ROUGEMONT, Denis de (1939). L'Amour et l'Occident. Paris : Plon.

RUSTIN, Jacques (1979). Le Vice à la mode. Paris : Ophrys.

SGARD, Jean (org.) (1996). Songe, illusion, égarement dans les romans de Crébillon. Grenoble : ELLUG, Université Stendhal.

SIEMEK, Andrzej (1981). La recherche morale et esthétique dans le roman de Crébillon fils. Oxford :

Voltaire Foundation. 
STENDHAL (1987). De l'Amour. Paris : Flammarion (1822).

STEWART, Philip (1973). Le Masque et la Parole : le langage de l'amour au XVIII siècle. Paris : Librairie José Corti.

STURM, Ernest (1970). Crébillon fils et le libertinage au XVIII ${ }^{e}$ siècle. Paris : Nizet.

VERSINI, Laurent (1968). Laclos et la tradition : essai sur les sources et la technique des Liaisons

dangereuses. Paris : Klincksieck.

VIART, Thierry (1999). La convention de l'amour-goût chez Claude Crébillon : genèse et perspectives.

Oxford : Voltaire Foundation.

WAGNER, Horst (1972). Crébillon fils : die erzählerische Struktur seines Werkes. München : Walter Fink Verlag.

\section{NOTES}

1. Il est curieux de remarquer l'identification, quelquefois abusive, courante à cette époque, entre les termes philosophe et libertin par opposition au croyant; quoique le libertinage soit un système philosophique, tout système philosophique de l'époque n'est pas, bien sûr, libertin.

2. Le Sylphe, Lettres de la Marquise, Les Égarements, La Nuit et le Moment, Le Hasard du coin du feu, Lettres de la Duchesse et même Les Heureux Orphelins (vu que, quoique l'action se passe dans le contexte anglais du début du XVIII ${ }^{e}$ siècle avec des personnages anglais, Chester, le protagoniste, a pour ambition de corrompre les mœurs anglaises selon le modèle du libertinage parisien).

3. Les trois contes de fées orientaux : Tanzaï et Néadarné (L'Écumoire), Le Sopha et Ah quel conte!; le roman épistolaire à plusieurs voix dont l'action se déroule du temps de Périclès, Lettres athéniennes, où le protagoniste, Alcibiade, tout comme Chester (Heureux Orphelins) et Versac (Égarements) est un dangereux libertin qui préfigure le couple pervers des Liaisons dangereuses.

\section{RÉSUMÉS}

Dans cet article je propose une lecture des récits de Claude Crébillon (1707-1777) comme une suite de chroniques du libertinage de «la bonne compagnie » du temps de la Régence de Philippe d'Orléans et du règne personnel de Louis XV. Injustement traité de licencieux, frivole, voire libertin, «Girgiro, l'entortillé », épithète que l'auteur doit à la tortuosité de son style, entreprend dans tous ses récits, d'un œil fin et ironique, une sorte de voyage philosophique à travers les différents jeux de pouvoir et de séduction, en présentant à son lecteur une chronique du libertinage mondain de son époque, dont il dévoile la fausseté dans tous ses états.

In this essay I would like to read the works written by the French novelist Claude Crébillon (1707-1777) as a series of worldly libertinage chronicles from the period of Phillipe d'Orléans' regency and the reign of Louis XV. Unjustly called licentious, frivolous and even libertine, 
"Girgiro, l'entortillé", epithet due to the tortuosity of the author's style, undertakes in all of his novels, from a sharp and ironic point of view, a kind of a philosophical journey through the different power and seduction games, presenting to his reader a worldly libertinage chronicle of his time disclosing all kind of its deceitfulness.

INDEX

Mots-clés : libertinage mondain dix-huitièmiste, Crébillon (Claude), roman-chronique Keywords : 18th Century worldly libertinage, Crébillon (Claude), chronicle novel

\section{AUTEUR}

ANA ALEXANDRA SEABRA DE CARVALHO

FCHS - Un. de l'Algarve/CLEPUL (Centro de Literaturas e Culturas Lusófonas e Europeias da Faculdade de Letras da Universidade de Lisboa, Pólo da Universidade do Algarve) aacarva[at]ualg.pt 